\title{
Oncogenic 3D genome conformations identify novel therapeutic targets in ependymoma
}

\section{Konstantin Okonechnikov}

Hopp Children's Cancer Center (KiTZ)

Aylin Camgoz

Hopp Children's Cancer Center (KiTZ)

Donglim Esther Park

UCSD

Owen Chapman

UCSD

Jens-Martin Hübner

Hopp Children's Cancer Center (KiTZ)

Abhijit Chakraborty

La Jolla Institute for Immunology

Meghana Pagadala

UCSD

Rosalind Bump

Salk Institute for Biological Studies

Sahaana Chandran

Salk Institute for Biological Studies

Katerina Kraft

Stanford University

Rocio Acuna-Hidalgo

10. Max Planck Institute for Molecular Genetics

\section{Derek Reid}

Arima Genomics, Inc

Edwin F. Juarez

UCSD

James T. Robinson

UCSD

Kristian W. Pajtler

German Cancer Research Center

Monika Mauermann

Hopp Children's Cancer Center NCT Heidelberg (KiTZ)

Till Milde 
German Cancer Research Center (DKFZ) https://orcid.org/0000-0002-7267-1052

\section{Nicole G. Coufal}

UCSD

\section{Michael Levy}

Rady Children's Hospital-San Diego

\section{Denise Malicki}

Rady Children's Hospital

\section{Shareef Nahas}

Rady Children's Hospital

\section{Matija Snuderl}

NYU Langone Health

John Crawford

Rady Children's Hospital

\section{Robert J. Wechsler-Reya}

Sanford Burnham Prebys Medical Discovery Institute https://orcid.org/0000-0002-7463-8352

\section{Stefan Mundlos}

Max Planck Institute for Molecular Genetics https://orcid.org/0000-0002-9788-3166

\section{Anthony Schmitt}

Arima Genomics

\section{Hannah Carter}

UCSD

\section{Kulandaimanuvel Antony Michaelraj}

Hospital for Sick Children

\section{Sachin A. Kumar}

The Hospital for Sick Children https://orcid.org/0000-0002-4853-3864

\section{Michael D. Taylor}

Sickkids Hospital

Jeremy Rich

Division of Regenerative Medicine, Department of Medicine, University of California San Diego

\section{Frank Buchholz}

TU Dresden

\section{Jill P. Mesirov}

University of California, San Diego

\section{Stefan M. Pfister}

Division of Pediatric Neurooncology, German Cancer Research Center (DKFZ)

\section{Ferhat Ay}

La Jolla Institute For Immunology https://orcid.org/0000-0002-0708-6914

Jesse R. Dixon

Salk Institute for Biological Studies https://orcid.org/0000-0002-6273-2181 


\section{Marcel Kool}

Division of Pediatric Neuro-oncology and German Cancer Consortium (DKTK), German Cancer Research Center (DKFZ), Heidelberg

Lukas Chavez ( $\square$ lukaschavez@health.ucsd.edu )

University of California, San Diego

\section{Biological Sciences - Article}

Keywords: ependymoma, tumorigenesis, oncogenic mechanisms

Posted Date: November 5th, 2020

DOI: https://doi.org/10.21203/rs.3.rs-88331/v1

License: (c) (i) This work is licensed under a Creative Commons Attribution 4.0 International License.

Read Full License 
Oncogenic 3D genome conformations identify novel therapeutic targets in

ependymoma.

4 Konstantin Okonechnikov ${ }^{1,2, *}$, Aylin Camgoz ${ }^{1,2,3,},{ }^{*}$, Donglim Esther Park ${ }^{4,5}$, Owen Chapman ${ }^{6}$, Jens-

5 Martin Hübner ${ }^{1,2}$, Abhijit Chakraborty ${ }^{7}$, Meghana Pagadala $^{6}$, Rosalind Bump ${ }^{8}$, Sahaana Chandran ${ }^{8}$,

6 Katerina Kraft $^{9}$, Rocio Acuna-Hidalgo ${ }^{10,11}$, Derek Reid ${ }^{12}$, Edwin F. Juarez ${ }^{6}$, James T. Robinson ${ }^{6}$,

7 Kristian W. Pajtler ${ }^{1,2,13}$, Monika Mauermann ${ }^{1,2}$, Till Milde ${ }^{1,13,14}$, Nicole G. Coufal ${ }^{15,18}$, Michael

8 Levy $^{16}$, Denise Malicki ${ }^{17}$, Shareef Nahas ${ }^{18}$, Matija Snuderl ${ }^{19,20}$, John Crawford ${ }^{21}$,

9 Robert J. Wechsler-Reya ${ }^{5,14,22}$, Stefan Mundlos ${ }^{10}$, Anthony Schmitt ${ }^{12}$, Hannah Carter ${ }^{6}$,

10 Kulandaimanuvel Antony Michealraj ${ }^{23}$, Sachin A. Kumar ${ }^{23}$, Michael D. Taylor ${ }^{23}$, Jeremy Rich ${ }^{4,5,24}$,

11 Frank Buchholz ${ }^{3,25,26}$, Jill P. Mesirov ${ }^{6,27}$, Stefan M. Pfister ${ }^{1,2,13}$, Ferhat Ay ${ }^{7,14}$, Jesse R. Dixon ${ }^{8}$,

12 Marcel Kool ${ }^{1,2,28, \#}$, Lukas Chavez 6 ,27,\#,+

1. Hopp Children's Cancer Center (KiTZ), Heidelberg, Germany;

2. Division of Pediatric Neurooncology, German Cancer Research Center (DKFZ) and German Cancer Consortium (DKTK), Heidelberg, Germany;

3. National Center for Tumor Diseases (NCT): German Cancer Research Center (DKFZ) Heidelberg, Faculty of Medicine and University Hospital Carl Gustav Carus, Technische Universität Dresden, Helmholtz-Zentrum Dresden-Rossendorf (HZDR), Dresden, Germany;

4. Division of Regenerative Medicine, Department of Medicine, University of California, San Diego, La Jolla, CA 92037, USA;

5. Sanford Consortium for Regenerative Medicine, 2880 Torrey Pines Scenic Drive, La Jolla, CA 92037, USA;

6. Division of Medical Genetics, Department of Medicine, University of California San Diego (UCSD), San Diego, USA;

7. Centers for Cancer Immunotherapy and Autoimmunity, La Jolla Institute for Immunology, La Jolla, CA, USA;

8. Peptide Biology Labs, Salk Institute for Biological Studies, La Jolla, CA, USA;

9. Center for Personal Dynamic Regulomes, Stanford University, Stanford, CA, USA ;

10. Max Planck Institute for Molecular Genetics, Berlin, Germany;

11. Institute for Medical Genetics and Human Genetics, Charité Universitätsmedizin Berlin, Berlin, Germany;

12. Arima Genomics, Inc. San Diego, CA, 92121; 
13. Department of Pediatric Oncology, Hematology and Immunology, Heidelberg University Hospital, Heidelberg, Germany;

14. CCU Pediatric Oncology, German Cancer Research Center (DKFZ) and German Cancer Consortium (DKTK), Heidelberg, Germany;

15. Department of Pediatrics, University of California, San Diego, San Diego, CA 92093, USA;

16. Neurosurgery, University of California San Diego - Rady Children's Hospital, San Diego, CA 92123, USA; 


\section{Abstract}

Ependymoma is a tumor of the brain or spinal cord. The two most common and aggressive molecular groups of ependymoma are the supratentorial RELA-fusion associated group and the posterior fossa ependymoma group A. In both groups, tumors occur mainly in young children and frequently recur after treatment ${ }^{1}$. Although the molecular mechanisms underlying these diseases have recently been uncovered, they remain difficult to target and innovative therapeutic approaches are urgently needed. Here, we use genome-wide chromosome conformation capture (Hi-C), complemented with CTCF (insulators) and $\mathrm{H} 3 \mathrm{~K} 27 \mathrm{ac}$ (active enhancers) ChIP-seq as well as gene expression and whole-genome DNA methylation profiling in primary and relapsed ependymoma tumors and cell lines to identify chromosomal rearrangements and regulatory mechanisms underlying aberrant expression of genes that are essential for ependymoma tumorigenesis. In particular, we observe the formation of new topologically associating domains ('neo-TADs') by intra- and inter-chromosomal structural variants, tumor-specific 3D chromatin complexes of regulatory elements, and the replacement of CTCF insulators by DNA hyper-methylation as novel oncogenic mechanisms in ependymoma. Through inhibition experiments we validated that the newly identified genes, including RCOR2, ITGA6, $L A M C 1$, and $A R L 4 C$, are highly essential for the survival of patient-derived ependymoma models in a disease subtype-specific manner. Thus, this study identifies potential novel therapeutic vulnerabilities in ependymoma and extends our ability to reveal tumor-dependency genes and pathways by oncogenic 3D genome conformations even in tumors that lack known genetic alterations.

\section{Main}

Tumors of the central-nervous system (CNS) are the most common cancers in children aged 0-14 years and a leading cause of death during childhood ${ }^{2-4}$. Intracranial ependymomas are segregated on the basis of anatomical location (supratentorial versus infratentorial or posterior fossa) and further divided by DNA methylation and expression profiling into distinct molecular groups that reflect differences in the age of onset, gender predominance, response to therapy, and genetic aberrations that drive the disease $^{1,5,6}$. The supratentorial RELA-fusion associated group is characterized by recurrent complex chromothripsis events in chromosome 11 that lead to different types of C11orf95-RELA fusion genes, which have been shown to drive tumorigenesis in this group of tumors ${ }^{7,8}$. In contrast, initial DNA sequencing studies showed an absence of recurrent mutations or gene fusions in posterior fossa ependymoma group A (PFA), suggesting that these tumors might be epigenetically driven ${ }^{5,7}$. Indeed, global loss of histone H3 lysine 27 trimethylation (H3K27me3), a histone modification associated with the negative regulation of gene expression, was identified as a marker for PFA tumors ${ }^{9}$. Recent studies 
have revealed that EZH inhibitory protein EZHIP (previously known as CXorf67), which is aberrantly

101 expressed in most PFA ependymomas, causes downregulation of H3K27me3 by inhibiting EZH2 in 102 the polycomb repressive complex $2(\mathrm{PRC} 2)^{10,11}$. The few PFA ependymomas that do not overexpress 103 EZHIP appeared to harbor K27M mutations in H3.1 or H3.3, which also inhibit EZH2. Furthermore, 104 gain of chromosome arm 1q, present in $25 \%$ of all PFA tumors, has been associated with a 105 particularly poor survival of PFA patients, but the underlying driver mechanism remains unknown ${ }^{10,12}$. 106 Since there are no small molecules available directly targeting the C11orf95-RELA fusions or EZHIP, 107 and since it is not yet known whether EZHIP alone drives tumorigenesis in PFA, a better understanding 108 of the tumor driving mechanisms and how they can be targeted is urgently needed. New insights into 109 the regulation of gene expression during normal and diseased human development have recently been 110 gained by analyzing 3D chromatin architectures ${ }^{13-15}$. Therefore, we have combined Hi-C with 111 complementary molecular profiling techniques of ependymoma tumors and cell lines to investigate 112 whether changes in intra- or inter- chromosomal DNA interactions in these tumors may lead to 113 activation of specific oncogenes and may identify novel targets and tumor dependencies (Figure 1a).

\section{The 3D genome organization of ependymoma tumors}

116 We have performed Hi-C ${ }^{16}$ followed by deep sequencing in 14 PFA and RELA ependymoma samples, 117 comprising eleven tumors (fresh frozen or FFPE) and three cell lines (Figure 1b, Suppl. Figure 1a). 118 Most samples were also analyzed by chromatin immunoprecipitation targeting the histone 119 modification H3K27ac, which is associated with active chromatin, followed by sequencing (ChIP-seq, $\mathrm{n}=9$ ), gene expression (RNA-seq, $n=11$ ), whole genome sequencing (WGS, $n=12$ ) and DNA methylation ( $n=14$, Suppl. Table 1). PFA and RELA ependymoma groups can be clearly distinguished using various molecular profiling techniques including DNA methylation ${ }^{17}$ (Suppl. Figure 1b).

123 Unsupervised clustering of the Hi-C data also clusters ependymoma tumors into the expected groups, 124 demonstrating pronounced group-specific 3D tumor genome conformations (Figure 1c, Suppl. 125 Figure 1c,d). By an integrative analysis of the Hi-C data, enhancers (defined by H3K27ac ChIP-seq enrichments), and gene expression, we observed that genes are generally expressed at higher levels when their promoters physically interact with enhancers or other gene promoters (Figure 1d). A large percentage ( $\sim 63-66 \%)$ of enhancer-associated genes (EAGs) previously predicted to be regulated by PFA or RELA enhancers ${ }^{18}$ can be confirmed to have chromatin interactions between gene promoters and enhancers by the analysis of subgroup-specific Hi-C maps (Figure 1e). For example, the Tenascin $C(T N C)$ promoter physically interacts with distal PFA enhancers (Figure 1f), which are positively correlated with TNC expression across a cohort of 24 tumors from six different intracranial ependymoma groups (Figure 1g, Suppl. Figure 1e). Overall, we found that more than twice as many 
134 genes as previously reported ${ }^{18}$ are potentially regulated by proximal and distal ependymoma enhancers 135 (Figure 1h, Suppl. Table 2,3). For example, the gene encoding Eukaryotic Translation Elongation 136 Factor 1 Alpha 2 (EEF1A2) interacts with subgroup specific enhancers (Figure 1i) and is specifically 137 upregulated in RELA -fusion associated tumors (Figure 1j). These and other regulatory dependencies 138 were not recognized in our previous $\operatorname{study}^{18}$, due to lack of data on chromatin interactions in 139 ependymoma samples and because TAD annotations from a fetal lung fibroblast cell line (IMR90) 140 were used instead (Suppl. Figure 1f,g).

Transcriptional activation of RCOR2 by neo-TAD formation in supratentorial RELA ependymoma

143 The formation of new topologically associating domains ('neo-TADs') through structural variation 144 was recently shown to have a critical role in gene dysregulation and oncogenesis ${ }^{19,20}$. To dissect the 145 effect of structural variants (SVs) in supratentorial RELA tumors on the potential formation of neo146 TADs, we used newly developed computational tools for the detection of SVs based on Hi-C data ${ }^{19,21}$ 147 (Suppl. Table 4). We first took a closer look at the C11orf95 and RELA gene loci, because it was 148 previously shown that the oncogenic C11orf95-RELA gene fusions are a result of chromothriptic 149 events on chromosome 11. As expected, the Hi-C data reproducibly detected structural variants at the 150 C11orf95 and RELA gene loci in the supratentorial RELA but not in PFA tumors (Figure 2a, Suppl. 151 Figure 2a). Furthermore, the Hi-C data captured extraordinarily complex rearrangements within 152 chromosome 11 in some RELA ependymoma samples (Figure 2b) and revealed that SVs are not 153 restricted to chromosome 11 but also include inter-chromosomal rearrangements (Figure 2c). In 154 particular, we observed intra- and inter-chromosomal structural variants in all RELA tumors, which 155 lead to the formation of neo-TADs placing the REST Corepressor 2 (RCOR2) gene in a new regulatory 156 environment (Figure 2d, Suppl. Figure 2b). RCOR2 is located $\sim 150 \mathrm{~kb}$ away of C11 1 rff 95 and has a 157 strong enhancer element upstream of its transcription start site that forms new DNA interactions with 158 the C11orf95 gene and other nearby enhancer elements by bridging the C11orf95-RELA breakpoint 159 (Figure 2e, Suppl. Figure 2c). By evaluating global Affymetrix gene expression array data ${ }^{1}$ across $^{2}$ 160 ependymoma groups, we found that RCOR2 expression is significantly upregulated in supratentorial 161 RELA relative to other ependymoma groups (p-value=1.71e-91, Figure 2f) and is highly correlated 162 with C11 lorf95 transcription $\left(\mathrm{R}=0.66\right.$, $\mathrm{p}$-value $=6.93^{-11}$, Suppl. Figure 2d). Interestingly, we also 163 identified RCOR2 as a hit in RELA cells in an independent shRNA screen (data not shown). To 164 validate the relevance of RCOR2 for tumor growth and maintenance, we performed shRNA-mediated 165 knock-down of RCOR2 expression in patient derived RELA and PFA ependymoma cell lines. We 166 observed that RCOR2 knockdown results in strongly reduced cell survival of supratentorial RELA and 167 to a lesser extent of PFA cell lines (Figure 2g-h). The on-target effect of shRNAs against RCOR2 was 
confirmed by western blot analysis of RCOR2 protein in RELA ependymoma cells (Suppl. Figure 2e). RCOR2 can form a protein complex with the histone de-methylase LSD1, also known as KDM1A, and other transcriptional co-repressors, including HDAC1/2 22 LSD1, HDAC1 and HDAC2 are all highly expressed across ependymoma subgroups and HDAC1/2 show pronounced RELA 172 ependymoma-specific expression (Suppl. Figures $\mathbf{2 f - h}$ ). Since there is no available compound against RCOR2, we reasoned that inhibition of other components of the RCOR2/LSD1/HDAC complex may still confer a therapeutic vulnerability for RELA ependymoma tumors. shRNA-mediated inhibition of LSD1 expression indeed leads to a significant depletion of RELA but not PFA ependymoma cells compared to scrambled shRNA (Figure 2i, Suppl. Figures 2i,j). Surprisingly, however, targeting the enzymatic activity of LSD1 with the LSD1 inhibitor ORY-1001 ${ }^{23}$ had no effect on cell survival using clinically accessible concentrations (Figure $\mathbf{2} \mathbf{j}$ ), suggesting that in this protein complex the protein rather than the enzymatic activity of LSD1 is important. In contrast, targeting the HDAC activity in

\section{PFA Ependymomas are dependent on integrin a6}

191 In all PFA tumors the Hi-C data revealed a 3D chromatin cluster that spatially links numerous 192 regulatory sequences and genes located more than 4 million base pairs apart on chromosome 2 (Figure 193 3a-c, Suppl. Figure 3a). To determine if this chromatin cluster is specific to PFA tumors, we analyzed 194 Hi-C data obtained from RELA ependymoma samples as well as normal human tissues and cell types 195 analyzed by the ENCODE and PsychENCODE consortia ${ }^{24,25}$. There was no sign of similar DNA interactions in the RELA and non-tumor samples, suggesting that this chromatin cluster is specific to PFA ependymomas (Figure 3a, Suppl. Figure 3a). By analyzing the expression of genes in this chromatin cluster in ependymoma and normal human brain samples, we observed that integrin $\alpha 6$ (ITGA6) expression, encoding the receptor of the extracellular matrix protein laminin, is significantly upregulated in PFA compared to RELA ependymoma and also normal human brain tissues (p-value: 
1.21e-114, Suppl. Figure 3b). As also reported previously ${ }^{1}$, gene-ontology analysis shows that 202 ITGA6-associated gene sets, such as extracellular matrix organization and positive regulation of cell 203 migration, are among the most highly enriched biological processes when comparing overall gene 204 expression profiles of PFA to other ependymoma groups (Suppl. Table 5). Recent genome-wide 205 CRISPR-Cas9 inhibition screens have revealed that ITGA6 is highly and specifically essential in PFA 206 ependymoma cell lines compared to glioblastoma (GBM) cell lines and fetal neural stem cells 207 (fNSCs) ${ }^{26,27}$ (Suppl. Figure 3c). In addition, we find that integrin $\beta 4$ (ITGB4), but not $\beta 1$ (ITGB1), is 208 significantly up-regulated in PFA compared to RELA ependymoma and normal brain samples (p209 values: 1.48e-119 and 1.42e-90, Suppl. Figure 3d), suggesting that the integrin $\alpha 6 \beta 4$ heterodimer is 210 the functional form relevant for PFA tumors. Based on these results, we hypothesized that PFA 211 ependymomas are locked in an oncogenic genomic topological configuration that activates ITGA6 212 transcription and drives the acquisition and maintenance of stemness. To test this hypothesis, we 213 performed CRISPR-Cas9 mediated ITGA6 knock-out in patient-derived PFA ependymoma cells by 214 cloning three different sgRNA sequences targeting ITGA6 into a lentiviral vector expressing Cas9 in 215 conjunction with GFP and subsequently transduced PFA ependymoma cells with the virus. As a result, 216 we observe that transduced PFA ependymoma cells (Figure 3d), but neither transduced RELA 217 ependymoma (Suppl. Figure 3e) nor glioblastoma cells (Suppl. Figure 3f), showed a gradual 218 decrease in cell proliferation over time, validating ITGA6 as an essential tumor-dependency gene 219 specific for PFA ependymomas.

\section{Transcriptional activation of $L A M C 1$ as a potential resistance mechanism in recurrent PFA}

223 Conventional copy-number variation (CNV) analyses previously showed 1q copy-number gains in a 224 subset of very aggressive and recurrent PFA ependymomas (Suppl. Figure 3g) ${ }^{10}$. By investigating 225 CNVs in PFA tumors, including primary and relapse tumors of the same patient, we observed frequent 226 increases in genomic instability in relapse tumors, while the 1q gain is maintained or emerges during 227 tumor progression (Suppl. Figure 3h). To elucidate the molecular mechanisms associated with 1q 228 gain, we systematically searched for SVs in all PFA ependymoma samples using the Hi-C data (Suppl. 229 Table 6). As expected, primary PFA EPNs have frequent DNA interactions within chromosomes 230 ('cis') and no DNA interactions indicative of recurrent structural variants (Suppl. Figure 3i). 231 However, we observed several complex inter-chromosomal DNA ('trans') interactions indicative of 232 structural variants in PFA ependymoma relapse tumors (Figure 3e, Suppl. Figure 3j). When 233 comparing SVs among the analyzed PFA ependymoma relapse samples, we observed a recurring event 234 that leads to an inversion of a $\sim 66 \mathrm{Mb}$ region of chromosome arm 1q into chromosome 8 (chr1-chr8 
in sample EPD210FH, Figure 3e), or into chromosome 3 (chr1-chr3 in sample RD-19-157, Suppl.

236 Figure 3j). In both cases, the breakpoints on chromosome 1q are located near the gene locus of laminin subunit $\gamma 1$ (LAMC1). Examination of the Hi-C data shows that both SVs led to the formation of neoTADs, which place $L A M C 1$ into new regulatory environments (Figure 3f,g). Inspecting RNA-seq expression data revealed that $L A M C 1$ is expressed almost three times higher in the two $1 \mathrm{q}+$ PFA ependymoma relapse tumors than in primary PFA ependymoma tumors (Figure 3h). The same pattern of increased $L A M C 1$ expression in PFA relapse cases was found in a larger Affymetrix ependymoma data cohort $^{1}$ (Figure 3i). By a direct comparison of three relapse tumors that developed within 18 years after the primary diagnosis of a PFA ependymoma patient, we observed upregulation of LAMC1 specifically in the third and fatal relapse tumor with marked chrlq gain (Suppl. Figure 3h,k). These results suggest that transcriptional activation of $L A M C 1$ by the formation of SV-induced neo-TADs is a resistance mechanism in recurrent $1 \mathrm{q}+$ PFA EPN tumors that potentially promotes proliferation and stemness by further enhancing an already excessive integrin signaling. Based on these results, we hypothesized that $L A M C 1$ expression is essential for the proliferation and growth of PFA ependymoma tumors. To test this hypothesis, we performed genetic (CRISPR-Cas9) inhibition experiments against LAMC1 and observed strongly reduced cell growth in the same PFA cell line EPD210FH that harbors the chr1-chr8 translocation (Figure 3j, Suppl. Fig. 3l) but not in RELA (Suppl. Figure 3m) ependymoma models.

\section{Hypermethylation disrupts CTCF binding in PFA ependymoma}

It has recently been shown that DNA methylation-mediated insulator dysfunction can lead to altered chromosomal topology thereby activating oncogenic programs (Figure 4a) ${ }^{28,29}$. Given the global loss of repressive $\mathrm{H} 3 \mathrm{~K} 27 \mathrm{me} 3^{10,11}$ and a previously reported DNA methylation phenotype in PFA ependymoma ${ }^{5}$, we hypothesized that similar molecular mechanisms may drive oncogene activation in this tumor type. Therefore, we analyzed 7 PFA $(n=4)$ and RELA $(n=3)$ tumors using Whole Genome Bisulfite Sequencing (WGBS) and CTCF ChIP-seq (Suppl. Table 1). As expected, genome wide CpG methylation is high in PFA and RELA ependymomas with low levels of methylation at functional regulatory elements, such as promoters, enhancers and insulators (Suppl. Figure 4a). By comparative analysis of DNA methylation at CTCF binding sites, we found that DNA hypermethylation replaces 2,387 CTCF binding sites in PFA tumors, but conversely is associated with the loss of only 178 CTCF binding sites in supratentorial RELA tumors (Figure 4b-d). The loss of CTCF binding through DNA hypermethylation is a predominant event in PFA ependymoma (Figure 4c) and can be associated with the formation of new enhancer-gene DNA loops and transcriptional activation of the target genes (Suppl. Table 7). To investigate whether such potential DNA methylation-mediated insulator 
dysfunctions can be linked to the transcriptional activation of genes essential for PFA ependymoma, we compared our results with those of a genetic inhibition screen in PFA cell lines ${ }^{26}$. Among others, we observed localized hypermethylation in PFA tumors associated with the loss of a CTCF binding site and the formation of DNA interactions between non-coding regulatory enhancer elements and the ADP Ribosylation Factor Like GTPase 4C (ARL4C) gene (Figure 4e-f). ARL4C transcription is significantly (p-value: 4.25e-55) upregulated in PFA tumors compared to other ependymoma groups (Suppl. Figure 4b) and is highly correlated with the activity of the enhancer elements that physically interact with the $A R L 4 C$ gene locus in PFA tumors (Figure 4g, Suppl. Figure 4c), but not in RELA tumors. It has been shown that ARL4C promotes migration, invasion and proliferation in colorectal and lung cancer ${ }^{30}$ and recent genome-wide CRISPR-Cas9 inhibition screens revealed that ARL4C is essential for the proliferation of PFA ependymoma compared to glioblastoma cell lines (Suppl. Figure 4d) ${ }^{26,27}$. By genetic (CRISPR-Cas9) inhibition experiments we validated that ARL4C is highly and specifically essential for the growth of PFA ependymoma (Figure 4h) compared to RELA ependymoma and glioblastoma models (Suppl. Figure 4e-f). These results not only provide additional evidence for the relevance of ARL4C in PFA ependymoma tumors, but also shed light on the various molecular mechanisms that potentially lead to oncogenic activation of gene expression through genome-wide epigenetic alterations.

\section{Discussion}

289 By investigating 3D ependymoma genomes using Hi-C, we have identified multiple oncogenic chromatin conformations and novel tumor-dependency genes, pathways and potential therapeutic targets in RELA and PFA ependymoma. We show that structural variants in supratentorial tumors not only lead to C11orf95-RELA fusion genes, but also result in the formation of new regulatory environments that are recurrently associated with the aberrant overexpression of $R C O R 2$. RCOR2 is 294 the scaffold protein in the CoREST complex that further contains LSD1 and HDAC1 and HDAC2. 295 The complex is associated with gene silencing and is known to play a role in cancer development ${ }^{31}$. 296 Here, we have shown that both RCOR2 and LSD1 expression is essential in RELA ependymoma, but not or to a lesser extent in PFA ependymoma, and that the cells are sensitive to HDAC1/2 inhibitors in line with our previous observations ${ }^{32}$. However, inhibition of the enzymatic activity of LSD1 had no effect. These results suggest that the activities of HDAC1/2 may be critical in regulating CoREST repressor functions in RELA ependymoma. Recent work in small cell lung cancer (and Merkel cell carcinoma) also implicated that disrupting the CoREST complex, but not the inhibition of LSD1's enzymatic activities is required for blocking cancer cell proliferation ${ }^{33}$. Further studies identifying the 
components of the CoREST complex and identifying drugs that can disrupt the complex will be 304 instrumental in developing an effective CoREST-targeted therapy for RELA ependymoma.

305 Furthermore, we have shown that PFA ependymomas are not only characterized by diminished histone methylation and increased acetylation at histone 3 lysine 27 (H3K27), as recently reported ${ }^{26}$, but also exhibit a tumor-specific 3D chromatin organization. Through targeting of ITGA6, a gene involved in a PFA-specific chromatin cluster, we demonstrate the importance of integrin signaling for maintained tumor growth, specifically in PFA tumors. ITGA6 has been described as a marker for cancer stem cells (CSCs) in several cancer types ${ }^{34-38}$, where disruption of ITGA6 function suppresses the CSC phenotype and the maintenance of stem cells ${ }^{34}$. Our results provide evidence for an epigenetic dysregulation event

312 that promotes integrin signaling and the acquisition of stemness in PFA ependymoma. The significance 313 of integrin signaling for PFA tumor progression is further promoted by the recurrent transcriptional 314 activation of LAMC1 in PFA relapse tumors which frequently harbor gains of chromosome 1q. 315 Although relapse tumors often show increased genomic instability, our Hi-C data showed for the first 316 time an unexpected complexity of intra- and inter- chromosomal rearrangements underlying some 317 chromosome-arm-wide copy number variations. Our results suggest that transcriptional activation of 318 LAMC1 by the formation of SV-induced neo-TADs is a potential resistance mechanism in recurrent $3191 \mathrm{q}+$ PFA EPN tumors that promotes proliferation and stemness by further enhancing already excessive 320 integrin signaling. For other tumor types, LAMC1 has already been shown to be involved in tumor 321 cell invasion and metastasis ${ }^{39}$. Thus, strategies that target integrin signaling, including ITGA6 and 322 LAMC1, may reveal new vulnerabilities and overcome resistance to therapy in the treatment of PFA 323 EPN relapse patients. Insulator dysfunction and oncogene activation by hypermethylation of CTCF 324 binding sites has recently been described in IDH mutant gliomas and in SDH-deficient gastrointestinal stromal tumors (GISTs) ${ }^{28,29}$. Here, we show that PFA ependymoma is another tumor type with a global epigenetic phenotype in which there is hypermethylation of CTCF binding sites and associated changes in genome topology. By genetic inhibition of $A R L 4 C$ in a PFA ependymoma model, we provide evidence that insulator dysfunction is a potential oncogenic mechanism in PFA ependymoma tumors and that tumor-dependency genes can be identified by 3D tumor genome profiling. Altogether, our study has identified several new group specific tumor dependencies in ependymoma, opening up avenues for potential novel therapeutic interventions that are highly needed in this disease, especially for RELA and PFA ependymoma. Our results will also be important for other (pediatric) cancers, especially those that relapse, where the drivers might be known, but where therapeutic options are scarce. Hi-C studies in these tumors may also reveal unknown tumor dependencies and new therapeutic targets. 
Figure 1: 3D tumor genome profiling identifies PFA and RELA specific chromatin conformations and ependymoma enhancer associated genes.

341 (a) Overview of the major results obtained by the application of genome-wide chromosome 342 conformation capture (Hi-C) in ependymoma brain tumors.

343 (b) Characteristics of ependymoma samples analyzed by Hi-C. One group of PFA ependymoma 344 samples has no apparent copy-number variants, while the other group of PFA samples exhibits 345 chromosome 1q gains associated with an unfavorable outcome.

346 (c) Unsupervised hierarchical clustering of PFA and RELA ependymoma tumors based on DNA 347 interactions (Hi-C) stratifies the samples into the expected molecular groups.

348 (d) Integrative analysis of enhancers (H3K27ac ChIP-seq), chromosome conformation (Hi-C) and 349 gene expression (RNA-seq) shows that genes are more strongly expressed when their promoters 350 physically interact with other promoters or with enhancers. Shown are tumors (3x PF-A, 3x RELA) 351 for which sample-matched H3K27ac ChIP-seq, RNA-seq, and HiC data are available. The center 352 line, box limits and whiskers indicate the median, upper/lower quartiles and $1.5 \times$ interquartile range 353 respectively. P-values from the bootstrap t-test are included.

354 (e) Re-evaluation of genes previously predicted to be regulated by PFA ependymoma enhancers 355 confirms that the promoter regions of approximately $66 \%$ of these genes $(n=1,028)$ physically 356 interact with PFA ependymoma enhancers. Similar results $(63 \%, n=1,229)$ are obtained for RELA 357 ependymomas.

358 (f) The $T N C$ promoter physically interacts with two distal enhancers (E1 and E2), whereby the 359 interaction with the more proximal enhancer E1 is much more pronounced in PFA than in RELA 360 tumors.

361 (g) TNC expression is positively correlated with the activity of the PFA-specific enhancer E1 362 (chr9:118146925-118163777), which is located 390kb upstream of the TNC transcription start site. 363 Here, a cohort of 24 tumors from six different intracranial ependymoma groups was examined.

364 (h) The integrative analysis of Hi-C, enhancer and gene expression data reveals that more than twice 365 as many genes as previously reported ${ }^{18}$ are regulated by proximal and distal ependymoma enhancers. 366 (i) The Hi-C data identifies a cluster of DNA interactions between a RELA-specific superenhancer 367 (SE) and the EEF1A2 gene. This regulatory dependency was not recognized previously because Hi368 C data obtained from IMR90 cells, which is commonly used as a reference, does not show any DNA 369 interactions and topologically associated domains at the EEF1A2 gene locus. 
(j) EEF1A2 expression is positively correlated with the RELA-specific SE

(chr20:62060923-62127745) highlighted in panel i. This regulatory dependency is further supported by the many RELA-specific DNA interactions observed in the Hi-C data.

\section{Figure 2: Transcriptional activation of RCOR2 by neo-TADs in RELA ependymoma}

(a) The Hi-C data reliably detect the structural variants that lead to the C11orf95-RELA fusion gene in supratentorial RELA-fusion associated tumors. Green boxes highlight SVs predicted by the computational methods applied.

(b) Chromothriptic rearrangements of chromosome 11 in a patient-derived RELA cell line (RELA BT165) visualized using Hi-C data.

(c) Structural variants in RELA tumors are not limited to chromosome 11 but also involve other chromosomes. Shown is an inter-chromosomal structural variant that includes chr11 and chr22 in tumor sample 7EP41.

(d) Reconstruction of the C11 lorf95-RELA breakpoint in a supratentorial tumor (4EP35) using Hi-C data reveals the formation of a neo-TAD that involves DNA interactions between RCOR2 and the C11orf95-RELA fusion gene. RNA-seq and H3K27ac ChIP-seq data of this sample are included as additional tracks.

(e) Genome browser visualization of the C11orf95 and RCOR2 genomic region shows RELA ependymoma-specific enhancers and DNA interactions.

(f) Boxplot of $R C O R 2$ gene expression across ependymoma groups using Affymetrix gene expression data $(n=393)$. The center line, box limits, whiskers and points indicate the median, upper/lower quartiles, $1.5 \times$ interquartile range and outliers, respectively. $R C O R 2$ is significantly upregulated in RELA tumors (anova p-val.: 1.71e-91). (g-i) shRNA time-course knockdown experiments in RELA (EP1NS) and PFA (EPD210FH) ependymoma cell lines using a scrambled control and two shRNA constructs each targeting either tagged and GFP positive cells are sorted by FACS. Results are normalized to day 0 and error bars represent SEM from two independent experiments.

(j-l) Dose response curves of single-compound treatment with ORY-1001 (j), Entinostat (k) or Corin (l) of RELA (EP1-NS) and PFA (EPD210FH) ependymoma spheroids over a 72-hour time-course using Celltiter-Glo cell viability assays. For each sample the results are presented as percentage of the Luminescence signal from control condition (i.e. water for ORY-1001 and DMSO for Entinostat and Corin as a vehicle). Data are presented as SEM from three independent experiments per tumor type. 


\section{Figure 3: PFA Ependymomas are dependent on integrin signaling}

406 (a) Hi-C DNA interaction matrices wherein a $\sim 5$ million base pair segment of chromosome 2 is 407 aligned along the diagonals shown for PFA (9EP1, left) and RELA (4EP53, middle) tumors and 408 normal cerebellum astrocytes (CAs, right). Off-diagonal signals indicate DNA interactions between 409 different genomic sites. The chromatin complex (highlighted by dashed circles) spatially links 410 several genes, including ITGA6, and various regulatory elements located more than 4 million base 411 pairs apart and is recurrently observed in all PFA ependymoma tumors, but in none of the other 412 samples analyzed.

413 (b) Hi-C DNA interactions of a PFA tumor (sample BT214) wherein the same $\sim 5$ million base pair 414 segment of chromosome 2 shown in panel (a) is aligned horizontally. Circles and dashed lines 415 highlight long-range DNA interactions.

416 (c) Genome browser view of the PFA-specific chromatin cluster shown in panels (a) and (b). The 417 included data tracks show long-range DNA interactions in PFA tumors ('Hi-C loops') as well as 418 gene expression and H3K27ac in RELA and PFA tumors.

419 (d) Genetic (CRISPR-Cass9) time-course knockout of ITGA6 in PFA ependymoma cells 420 (EPD210FH) using an unspecific control and three individual sgRNA constructs. All constructs are 421 GFP tagged and GFP positive cells are sorted by FACS. Results are normalized to day 0 and error 422 bars represent SEM from two independent experiments.

423 (e) The conventional copy number profile of a PFA relapse sample (EPD210FH) shows high 424 genomic instability including gain of chromosome arm 1q (presented vertically on the left). The 425 genome-wide DNA interaction (Hi-C) map obtained from the same sample identifies complex inter426 chromosomal structural variants including an inversion that involves chrlq and chr8. These trans427 SVs reveal the complexity of genomic rearrangements underlying some copy-number gains and 428 losses observed by common copy number variation analyses.

429 (f) Re-construction of the structural variant that involves chr1q and chr8 in the PFA relapse sample 430 EPD210FH using Hi-C data. This structural variant results in the formation of a neo-TAD that places 431 the LAMC1 gene locus in a new regulatory environment.

432 (g) Re-construction of the structural variant that involves chr1q and chr3 in the PFA relapse tumor 433 RD-19-157 using Hi-C data obtained from FFPE material. This structural variant also results in the 434 formation of a neo-TAD that places the LAMC1 gene locus in a new regulatory environment.

435 (h) Boxplot of RNA-seq expression analysis revealed that $L A M C 1$ is expressed almost three times 436 higher in the two 1q+ PFA ependymoma relapse tumors than in primary PFA tumors, suggesting that 437 the transcriptional activation of $L A M C 1$ by the formation of SV-induced neo-TADs is a common 
resistance mechanism in recurrent 1q+ PFA EPN tumors. The center line, box limits, whiskers and

439 circles indicate the median, upper/lower quartiles, $1.5 \times$ interquartile range and samples, respectively.

440 (i) Boxplot of Affymetrix gene expression data shows that LAMC1 tends to be upregulated in a

441 larger cohort of relapse PFA in comparison to primary PFA ependymoma tumors. The center line,

442 box limits, whiskers and points indicate the median, upper/lower quartiles, $1.5 \times$ interquartile range

443 and outliers, respectively.

444 (j) Genetic (CRISPR-Cass9) time-course knockout of LAMC1 in PFA ependymoma cells

445 (EPD210FH) using an unspecific control and three individual sgRNA constructs. All constructs are

446 GFP tagged and GFP positive cells are sorted by FACS. Results are normalized to day 0 and error

447 bars represent SEM from two independent experiments.

\section{Figure 4. Hypermethylation replaces CTCF binding sites in PFA ependymoma.}

450 (a) Proposed mechanism of epigenetic oncogene activation in PFA ependymoma tumors. Top: The 451 oncogene is separated from an enhancer by a CTCF insulator, which forms a topological barrier.

452 Below: The CTCF insulator is replaced by DNA methylation so that the enhancer can contact the 453 oncogene and enhances its expression.

454 (b) The volcano plot shows significant differential CTCF binding sites between PFA and RELA 455 ependymoma tumors (min $p$-value: 0.1 ). CTCF binding sites significantly hypermethylated in PFA 456 relative to RELA tumors are marked in orange (min q-value: 0.05 ).

457 (c) Comparison of CTCF binding strength (CTCF ChIP-seq, $\mathrm{x}$-axis, min $p$-value 0.1, min fold 458 change: 0.5 ) and DNA methylation (WGBS, y-axis, min $q$-value: 0.05 , min difference: 0.1 ) at CTCF 459 binding sites that show significant differences between PFA and RELA ependymoma tumors.

460 (d) Heatmap of WGBS-derived DNA methylation at the 300 most significant CTCF binding sites 461 lost in three PFA (left) and three RELA (right) ependymoma tumors. The heatmaps and the 462 composite panels on top show that CTCF binding sites are commonly replaced by DNA methylation 463 in PFA tumors.

464 (e) Genome browser visualization of PFA ependymoma-specific DNA loops that associate two PFA 465 enhancers (E1 and E2) with the $A R L 4 C$ gene locus located 3,520 kbp away from the $A R L 4 C$ 466 transcription start site.

467 (f) WGBS-derived DNA methylation and CTCF ChIP-seq data from PFA and RELA ependymoma 468 tumors show that a CTCF binding site separating the ARLC4 gene from the E1 and E2 PFA 469 enhancers is replaced by DNA methylation in PFA tumors. 
(g) $A R L 4 C$ gene expression is positively correlated with the activity of enhancer E2

(chr2:237763494-237764993) across a cohort of 24 ependymoma tumors form six different

472 intracranial ependymoma groups.

473 (h) Genetic (CRISPR-Cass9) time-course knockout of $A R L 4 C$ in PFA ependymoma cells

474 (EPD210FH) using an unspecific control and three individual sgRNA constructs. All constructs are

475 GFP tagged and GFP positive cells are sorted by FACS. Results are normalized to day 0 and error 476 bars represent SEM from two independent experiments.

\section{Supplementary Figure 1}

480 (a) The number of DNA contacts obtained in the individual ependymoma samples as a quality 481 measure for the Hi-C data.

482 (b) The tSNE dimensionality reduction visualization and unsupervised clustering of DNA 483 methylation data from a cohort of ependymoma samples $(n=1,182)$ separates PFA and RELA 484 ependymomas into separate groups. The samples analyzed in this study by Hi-C are highlighted in 485 red and orange. Blocks marked with an asterisk show a magnification of selected samples, for better 486 visualization.

487 (c) Proportions of topologically associated domains (TADs) shared between ependymoma tumors. 488 The comparison is performed among PFA $(n=3)$ and RELA $(n=3)$ ependymoma tumors that were 489 also included in our previous enhancer-mapping study ${ }^{18}$. Hi-C data from two normal cerebellum 490 astrocytes provided in the ENCODE database ${ }^{40}$ were included as controls. The mean proportion of 491 common TADs is 0.569 and the greatest similarity is found in TADs from samples of the same group 492 (PFA, RELA and cerebellar astrocytes), while the common TAD proportions in the individual 493 sample groups are smaller.

494 (d) Numbers of common TADs between EPN PFA (n=3) and RELA (n=3) samples from enhancer 495 landscape study. The highest number of same TADs is among all samples.

496 (e) TNC expression is positively correlated across a cohort of 24 tumors from six different 497 intracranial ependymoma groups with the activity of the PFA ependymoma-specific enhancer E2 498 (chr9:118275447-118288653) located upstream of the TNC transcription start site.

499 (f-g) Hi-C data visualization of a genomic region that includes the EEF1A2 gene. The association 500 between a RELA ependymoma super enhancer (SE) and the up regulation of EEF1A2 transcription 501 was not detected previously due to the lack of annotated TADs at this gene locus in the Hi-C data 502 from IMR90 samples. TADs derived from IMR90 (blue), RELA (RELA BT, red), and PFA 503 (EPD210FH, orange) ependymoma samples are visualized in boxes. 


\section{Supplementary Figure 2}

506 (a) Visualization of the Hi-C data at the extended C11orf95 and RELA gene locus in PFA 507 ependymoma tumors shows the absence of structural variants.

508 (b) Reconstruction of the C11 1orf95-RELA breakpoint in RELA ependymoma tumors (7EP41 and 509 11EP22) using Hi-C data reveals the formation of a neo-TAD that involves DNA interactions 510 between RCOR2 and the C11orf95-RELA fusion gene. RNA-seq, CTCF and H3K27ac ChIP-seq data 511 of these samples are included as additional tracks.

512 (c) Chr11 genome browser visualization showing DNA loops that span the C11orf95 and RELA gene 513 loci.

514 (d) Correlation between the expression of $R E L A$ and $R C O R 2(\mathrm{R}=0.663, p$-val=6.93e-11) in RELA 515 ependymoma tumors $(\mathrm{n}=76)$ profiled by Affymetrix gene expression arrays.

516 (e) Knock-down of $R C O R 2$ expression in RELA ependymoma cells. Western blots show protein 517 levels 4 days post infection with indicated shRNAs. B-actin is used as a loading control.

518 (f-h) Boxplots of $L S D 1, H D A C 1$ and $H D A C 2$ gene expression across ependymoma subgroups using 519 Affymetrix gene expression data ( $\mathrm{n}=393$, anova $\mathrm{p}$-values: 9.77e-15, 1.02e-27, 1.20e-14). The center 520 line, box limits, whiskers and points indicate the median, upper/lower quartiles, $1.5 \times$ interquartile 521 range and outliers, respectively.

522 (i) Knock-down of $L S D 1$ expression in ependymoma cells. Western blots show protein levels 4 days 523 post infection with indicated shRNAs. B-actin is used as a loading control.

524 (j) shRNA time-course knockdown experiments in a PFA ependymoma cell line (EPD210FH) using 525 a scrambled control and two shRNA constructs targeting LSD1. All constructs are GFP tagged and 526 GFP positive cells are sorted by FACS. Results are normalized to day 0 and error bars represent 527 SEM from two independent experiments.

528 (k-l) Dose response curves of the HDAC8 inhibitor PCI-34051 (k) and the HDAC 6/10 inhibitor 529 Tubastatin (l) treatment of RELA (EP1NS, red) and PFA (EPD210FH, orange) ependymoma 530 spheroids over a 72-hour time-course using Celltiter-Glo cell viability assays. For each sample, the results are presented as percentage of the Luminescence signal from control condition (DMSO as a

\section{Supplementary Figure 3}

535 (a) DNA interaction matrices derived from $\mathrm{HiC}$ data wherein a $\sim 5$ million base pair segment of 536 chromosome $2(172,407,031-177,907,030 \mathrm{bp})$ is aligned along the diagonals. Off-diagonal signals indicate DNA interactions between different genomic sites. The chromatin complex at the ITGA6 
locus recurrently forms in all PFA EPN tumors analyzed (shown for samples 9EP1, 9EP9, and 7EP18 in the top row). This chromatin complex is not present in RELA ependymoma tumors (middle row). Moreover, normal human cell types analyzed by the ENCODE and PsychENCODE consortia, such as cerebellar astrocytes (CA), neural progenitor cells (NPCs) and embryonic fibroblasts (IMR90, bottom row), do not show signs of similar DNA interactions, suggesting that this chromatin complex is characteristic for PFA ependymoma tumors.

(b) Differential gene expression analysis of Affymetrix array data identified ITGA6 as significantly (FDR $<0.01$, two-sided Wilcoxon test) upregulated in PFA compared to RELA ependymoma tumors and normal brain samples ( $n=200$ PFA, $n=76$ RELA, and $n=225$ normal human brain samples). In the boxplot the center line, box limits, whiskers and points indicate the median, upper/lower quartiles, $1.5 \times$ interquartile range and outliers, respectively.

(c) ITGA6 has been observed as an essential gene specifically in PFA ependymoma cell lines compared to glioblastoma stem cells (GSCs) and fetal neural stem cells (fNSCs) in a published CRISPR-Cas9 knock-out screen ${ }^{26}$. In the boxplot the center line, box limits and whiskers indicate the median, upper/lower quartiles and $1.5 \times$ interquartile range, respectively.

(d) Expression of two alternative ITGA6 heterodimer partner proteins ITGB1 and ITGB4. While $I T G B 1$ and ITGB4 are highly expressed in PFA and RELA ependymoma tumors compared to normal brain samples, only ITGB 4 is significantly upregulated (FDR $<0.01$, two-sided Wilcoxon test) in PFA compared to RELA ependymoma tumors, suggesting that the integrin $\alpha 6 \beta 4$ heterodimer is the functional form relevant for PFA ependymoma tumors ( $n=200$ PFA, $n=76$ RELA, and $n=225$ normal human brain samples). In the boxplots the center line, box limits, whiskers and points indicate the median, upper/lower quartiles, $1.5 \times$ interquartile range and outliers, respectively.

(e) Genetic (CRISPR-Cas9) time-course knockout of ITGA6 in RELA ependymoma cells (EP1-NS) using an unspecific control and three individual sgRNA constructs. All constructs are GFP tagged and GFP positive cells are sorted by FACS. Results are normalized to day 0 and error bars represent SEM from two independent experiments.

(f) Genetic (CRISPR-Cas9) time-course knockout of ITGA6 in glioblastoma (GBM2) cells using an unspecific control and three individual sgRNA constructs. All constructs are GFP tagged and GFP positive cells are sorted by FACS. Results are normalized to day 0 and error bars represent SEM from two independent experiments.

(g) Example of a copy number profile of a PFA ependymoma tumor (16EP7) harboring a 1q gain. The CNV profile was obtained from DNA methylation array data.

(h) Copy number profiles of a primary and three relapse PFA ependymoma tumors from the same patient (RD-19-157). The CNV profiles were obtained from DNA methylation array data. 
572 (i) Visualization of Hi-C data obtained from a primary PFA ependymoma tumor (16EP7) harboring a

573 1q gain shows frequent DNA interactions within chromosomes but no DNA interactions indicative

574 of inter-chromosomal structural variants.

575 (j) The conventional copy number profile of a PFA relapse sample (RD-19-157) shows high

576 genomic instability including gain of chromosome arm 1q (presented vertically on the left). The

577 genome-wide DNA interaction (Hi-C) map obtained from the same sample identifies complex inter-

578 chromosomal structural variants including an inversion that involves chr1q and chr3.

579 (k) Expression of $L A M C 1$ in the three relapse tumors of patient RD-19-157 shows up-regulation of

$580 L A M C 1$ in the third relapse tumor that harbors a 1q gain.

581 (l) Western blots show efficacy of sgRNAs targeting LAMC1 and control sgRNA in EPD210FH

582 cells. Mixture of different clones of infected EPD210FH cells are used to obtain protein extracts at

583 day 5 post infection with indicated sgRNAs. B-actin is used as a loading control.

584 (m) Genetic (CRISPR-Cas9) time-course knockout of LAMC1 in RELA ependymoma (EP1-NS)

585 cells using an unspecific control and three individual sgRNA constructs. All constructs are GFP

586 tagged and GFP positive cells are sorted by FACS. Results are normalized to day 0 and error bars

587 represent SEM from two independent experiments.

\section{Supplementary Figure 4}

590 (a) Mean methylation of PFA and RELA ependymoma tumors at promoters, enhancers and CTCF

591 binding sites. The center line, box limits, whiskers and points indicate the median, upper/lower

592 quartiles, $1.5 \times$ interquartile range and outliers, respectively.

593 (b) Boxplot showing $A R L 4 C$ gene expression across ependymoma subgroups (Affymetrix gene

594 expression data for $\mathrm{n}=393$ ependymoma tumors). The center line, box limits, whiskers and points

595 indicate the median, upper/lower quartiles, $1.5 \times$ interquartile range and outliers, respectively. $A R L 4 C$

596 is significantly upregulated in PFA compared to the other ependymoma types (anova p-val.: 4.25e$59755)$.

598 (c) Positive correlation of $A R L 4 C$ gene expression and the activity of enhancer E1

599 (chr2:237545700-237546199) across a cohort of 24 ependymoma tumors form six different 600 intracranial ependymoma groups.

601 (d) $A R L 4 C$ has been observed as an essential gene in PFA ependymoma cell lines in a published

602 CRISPR-Cas9 knock-out screen ${ }^{26}$. In the boxplot the center line, box limits and whiskers indicate 603 the median, upper/lower quartiles and 1.5× interquartile range, respectively.

604 (e) Genetic (CRISPR-Cas9) time-course knockout of $A R L 4 C$ in RELA ependymoma (EP1-NS) cells 605 using an unspecific control and three individual sgRNA constructs. All constructs are GFP tagged 
606 and GFP positive cells are sorted by FACS. Results are normalized to day 0 and error bars represent

607 SEM from two independent experiments.

608 (f) Genetic (CRISPR-Cas9) time-course knockout of $A R L 4 C$ in glioblastoma (GBM2) cells using an 609 unspecific control and three individual sgRNA constructs. All constructs are GFP tagged and GFP

610 positive cells are sorted by FACS. Results are normalized to day 0 and error bars represent SEM

611 from two independent experiments.

612

\section{Supplementary Tables}

614

615 Supplementary Table 1: Cohort of ependymoma tumor samples and available data types.

617 Supplementary Table 2: Enhancer associated genes supported by DNA loops in PFA ependymoma.

619 Supplementary Table 3: Enhancer associated genes supported by DNA loops in RELA

620 ependymoma.

622 Supplementary Table 4: Structural variants in RELA ependymoma samples as identified by

623 (a) hicBreakFinder. No additional filter was applied. (b) HiC SV/trans with adjusted filtering.

625 Supplementary Table 5: DAVID Gene Ontology analysis results for differentially expressed genes specific for PFA compared to other ependymoma subgroups.

Supplementary Table 6: Structural variants in PFA ependymoma samples as identified by

(a) hicBreakFinder. No additional filter was applied. (b) HiC SV/trans with adjusted filtering.

Supplementary Table 7: Enhancer-associated genes supported by DNA loops that are potentially formed due to the replacement of CTCF binding sites by DNA methylation in PFA ependymoma

Supplementary Table 8: Overview of shRNA and sgRNA oligo sequences applied in experimental validation.

638 Supplementary Table 9: Drugs selected for experimental validation 
640 Supplementary Table 10: Overview of antibodies applied in experimental validation.

\section{Acknowledgements}

644 We are thankful to David T. Jones for providing pediatric patient-derived SU-pcGBM2 cells. This 645 work is supported by a Hannah's Heroes St. Baldrick's Scholar Award (LC) and funding from the 646 NIH National Institute of Neurological Disorders and Stroke Institute R21 NS116455 (LC), the NIH 647 National Cancer Institute U01 CA217885 (JPM), U01 CA184898 (JPM), U24 CA210004 (JPM and 648 JTR); the NIH National Institute of General Medical Sciences R01GM074024 (JPM); and the NIH 649 National Library of Medicine T15LM011271 for OC. The pediatric brain profiling at NYU in M.S. 650 laboratory was in part supported by grants from the Friedberg Charitable Foundation and the Making 651 Headway Foundation. Work in the laboratory of J.R.D. was supported by a NIH Early Independence 652 Award (DP5 OD023071).

\section{Contributions}

K.O., A.C., M.K. and L.C. prepared the manuscript and figures. K.O., O.C., A.C., M.P. J.T.R., E.F.J. and A.S. performed data analysis and visualization. A.C., D.E.P., M.M., and J.M.H. performed experimental validations, N.G.C., M.L., D.M., S.N., and M.S. processed and analyzed tumor material, R.B., S.C., K.K., R.A.H. and D.R. generated Hi-C libraries from frozen and FFPE tumor material and cell lines, K.W.P., T.M., S.M., A.S., H.C., J.C., R.W.R., K.A.M., S.A.K., M.D.T., F.B., J.R., J.P.M., S.M.P., F.A. and J.R.D. contributed to the study design and data interpretation. L.C. 


\section{Chromosome conformation capture}

$\mathrm{Hi}-\mathrm{C}$ on frozen tumor tissue sample was carried out using protocols previously described for tissue $\mathrm{Hi}$ $\mathrm{C}$ experiments ${ }^{41}$. In brief, frozen tissues are pulverized using a mortar and pestle kept cold on a bed of dry ice into a fine powder. The tissue powder was then transferred to a $15 \mathrm{~mL}$ conical tube containing

$6735 \mathrm{mLs}$ of DPBS and fixed with $2 \%$ formaldehyde for 10 minutes. The fixation was quenched by 674 addition of $0.2 \mathrm{M}$ Glycine. The fixed tissue was pelleted by centrifugation, washed 1x with DPBS, and 675 then flash frozen until ready for further processing.

676 For Hi-C experiments, the fixed frozen tissue pellets were first resuspended in $3 \mathrm{mLs}$ of lysis buffer (10mM Tris-HCl pH 8.0, 5mM CaCl $2,3 \mathrm{mM} \mathrm{MgAc}$, 2mM EDTA, 0.2mM EGTA, 1mM DTT, 0.1mM PMSF, 1X Complete Protease Inhibitors). The sample was transferred to an M-tube and dissociated using a GentleMACS Tissue dissociator (Miltenyi) using the "Protein M-tube" setting. The sample was removed from the M-tube into a $50 \mathrm{~mL}$ conical. The M-tube was washed with $3 \mathrm{mLs}$ of lysis buffer with $0.4 \%$ Triton $\mathrm{X}-100$ added, and this wash was combined with the original $3 \mathrm{mLs}$ of sample for a total volume of $6 \mathrm{mLs}$ with final concentration of $0.2 \%$ Triton $\mathrm{X}-100$. The sample was then passed through a $40 \mu \mathrm{M}$ cell strainer. The strainer was washed with an additional $2 \mathrm{mLs}$ of lysis buffer with $0.2 \%$ Triton $\mathrm{X}-100$. The sample was then centrifuged and washed with $1 \mathrm{~mL}$ of lysis buffer with $0.2 \%$ Triton X-100. After centrifugation, the sample was resuspened in $0.5 \%$ SDS and processed with previously described in situ Hi-C method ${ }^{42}$ using the MboI enzyme. Libraries were prepared using the Illumina TruSeq LT sequencing adaptors. Initial QC sequencing was first performed on a MiSeq to assess library quality, and if sufficient, was subject to production scale sequencing on the HiSeq X or

\section{Chromosome conformation capture from FFPE material}

692 Hi-C experiments on FFPE material were carried out by Arima Genomics, Inc (San Diego, CA). 693 Dewaxed and re-hydrated FFPE tissue was used as input to a modified version of the Arima-HiC Kit 694 protocol. After the Arima-HiC protocol, Illumina-compatible sequencing libraries were prepared by 695 shearing the proximally ligated DNA and then size-selecting DNA fragments using SPRI beads. The 696 size-selected fragments containing ligation junctions were enriched using Enrichment Beads (provided 697 in the Arima-HiC Kit), and converted into Illumina-compatible sequencing libraries using the Swift 698 Accel-NGS 2S Plus kit (P/N: 21024) reagents. After adapter ligation, DNA was PCR amplified and 
699 purified using SPRI beads. The purified DNA underwent standard QC (qPCR and Bioanalyzer) and

700 sequenced on the NovaSeq following manufacturer's protocols.

\section{Hi-C data processing}

703 The sequencing reads alignment to hg19 human genome reference and chromatin contacts calling was 704 performed using HiCPro 2.9.0 toolkit ${ }^{43}$ allowing the bin sizes 5,10,50,100,250 and $500 \mathrm{Kbp}$. Main 705 visualization and normalized full contacts extraction was performed with JuiceBox v0.7.5 toolkit ${ }^{44}$. 706 Per sample loop calling was applied from FitHiC v2.0.6 method ${ }^{45}$ on bin sizes $5 \mathrm{Kbp}$ with maximum 707 distance between bins $50 \mathrm{Mbp}$. TAD calling was performed based on $50 \mathrm{Kbp}$ bins resolution using 708 TopDom tool ${ }^{46}$.

\section{Unsupervised clustering of $\mathrm{Hi}-\mathrm{C}$ data}

711 Hi-C data processing produced interaction matrices in .juicebox format for 3 RELA and 8 PFA 712 ependymoma tumors. For each tumor, unsupervised clustering features were computed using the 713 Eigenvector utility from the Juicer Tools analysis toolkit. ${ }^{47}$ Briefly, the Eigenvector utility computes $714 \mathrm{~A} / \mathrm{B}$ compartments as the first principal component of the Pearson correlation matrix of each intra715 chromosomal contact matrix. ${ }^{48} \mathrm{~A} / \mathrm{B}$ compartments were computed using Knight-Ruiz normalization 716 at $1 \mathrm{Mb}$ resolution. Unsupervised hierarchical clustering was performed on these features using Pearson 717 correlation distance and average linkage, using the heatmap.2 function from gplots R package.

\section{Genes and enhancers connections via loops}

720 The ChIP-seq derived enhancer signals along with genomic locations of group-specific enhancers and 721 normalized RNA-seq gene expression profiles from epenymoma tumors cohort $(\mathrm{n}=25)$ were obtained 722 from published materials of the corresponding study ${ }^{18}$. Genome was fragmented into $5 \mathrm{Kbp}$ bins and 723 output from FitHiC loop calling tool was used to find contacts between genes and enhancers. For this 724 purpose the genes were assigned to bins based on the location of transcription start site (TSS, 2500 $725 \mathrm{Kbp}$ upstream and downstream of the gene stat loci), while enhancers based on the overlap. Loop 726 boundary correspondence was assigned to gene and/or group-specific enhancer lying either within the 727 bin or in the closest upstream/downstream bin. Enhancer associated gene was considered to be 728 supported by loop if the TSS of it was lying in one loop anchor while enhancer in the other. Correlation 729 analysis was performed based on the usage of updated InTAD package ${ }^{49}$ v1.9.2.

\section{Gene expression analysis}


The global ependymoma tumor gene expression data integration was performed based on the usage of corresponding R2 platform materials with focus on Affymterix dataset from combined ependymoma tumors cohort with integration of normal brain tissues $(n=618)$. Major of these ependymoma tumor Affymetrix materials were obtained from the corresponding main study ${ }^{1}$ (GEO: GSE64415) with additional external inclusions (GEO: GSE50161, GSE50385, GSE21687, GSE3526). The gene ontology analysis was performed using DAVID tool ${ }^{50}$ based on the usage of differentially expressed genes between PFA and other ependymoma groups achieved with R2 platform from the EPN global Affymetrix dataset. The RNA-sequencing materials from target EPN cohort samples were analyzed as previously described ${ }^{18}$.

\section{Structural variants (SV) discovery from Hi-C data}

743 SV discovery from Hi-C data was performed using two independent toolkits. The first toolkit, 744 hicBreakFinder (https://github.com/dixonlab/hic breakfinder), was adjusted for the usage on hg19 745 human genome reference with taking into account additional filtering lists of false positives obtained 746 from external cohorts ${ }^{19}$. Shortly, the tool scans for abrupt shifts in chromosomal connections in order 747 to find possible outliers representing inter/intra-chromosomal events based on the selected threshold $748(\mathrm{t}=0.6)$ and reports them in resolutions $1 \mathrm{Mb}, 100 \mathrm{~Kb}$ and $10 \mathrm{~Kb}$. Final combined result contains the 749 highest resolution for detected SV. The second toolkit, Hi-C structural variant discovery or HiCsv, 750 consists of two parts and was adjusted for the usage of hg38 genome as the most up-to-date reference 751 genome. First part of this toolkit, HiCtrans ${ }^{21}$, focuses on inter-chromosomal translocations: it scans 752 the inter-chromosomal contact matrices over multiple $\mathrm{Hi}-\mathrm{C}$ resolutions for each possible pair of 753 chromosomes from a given sample and predicts candidate SVs based on the changepoint analysis using 754 binary segmentation. The intra-chromosomal translocations are also detected in this toolkit based on 755 the dual pattern of off-diagonal enrichment and diagonal depletion of chromatin interactions in a Hi$756 \mathrm{C}$ map across genomic regions. HiCsv detects enrichment of interactions through FitHiC2 algorithm ${ }^{45}$ 757 and uses an insulation score-based estimation (similar to TAD finding ${ }^{51}$ ) to identify depletion in 758 interaction frequency. Finally, it applies a density-based clustering of enriched Hi-C interactions with high insulation scores to discover structural variants.

\section{CTCF ChIP-sequencing}

762 ChIP-sequencing procedure was prepared and performed as previously described ${ }^{18}$. Shortly, ChIP 763 flash-frozen for ependymoma tumours was performed using $5 \mu \mathrm{g}$ CTCF antibody per ChIP Active 764 Motif. Enriched DNA was quantified and barcoded. Following library amplification, DNA fragments 765 were sequenced using Illumina HiSeq 2000 100-bp paired-end sequencing. 


\section{CTCF ChIP-seq data analysis}

768 Reads alignment was performed to hg19 reference with BWA v0.5.10 $0^{52}$. Duplicate alignments were 769 removed using Picard (http://broadinstitute.github.io/picard). Peak calling was performed using Macs $770 \mathrm{v} 1.4^{53}$. Differential RELA peaks between EPN PFA and RELA were detected suing DiffBind R 771 package ${ }^{54}$ with min adjusted p-value limit 0.05 .

\section{Whole genome bisulfite sequencing (WGBS)}

774 WGBS procedure was prepared and performed as previously described ${ }^{55}$. Shortly, $5 \mu \mathrm{g}$ of genomic DNA were sheared using a Covaris device. After adaptor ligation, DNA fragments were isolated and bisulphite converted using the EZ DNA Methylation kit (Zymo Research). PCR amplification of the fragments was performed followed by library aliquots pooling. Sequencing was performed Illumina HiSeq 2000 machine.

\section{WGBS data analysis}

Initial reads processing was performed using methylCtools v0.9.4 as previously described ${ }^{55}$. Differentially methylated regions were detected using metilene v0.2.6 tool ${ }^{56}$ with min adjusted pvalue limit 0.05 . Combined visualization of the methylation profiles within CTCF target regions was performed using the EnrichedHeatmap R package. Search of target loops was performed based on the presence of overlapping the DMRs with differential CTCF peaks within.

\section{Cell culture}

HEK293T cells (CRL-1273, American Type Culture Collection) were cultured as previously described (citation). EPD210FH cells were grown in NeuroCult NS-A Basal Medium (STEMCELL Technologies) supplemented with NeuroCult Proliferation Supplement (STEMCELL Technologies), 2mM L-glutamine 1\% Penicillin/Streptomycin, $75 \mathrm{ng} / \mathrm{ml}$ bovine serum albumin (BSA) and 20ng/ml of 792 EGF (Peprotech) and FGF-basic (Peprotech). EP1NS cells were grown in Neurobasalmedium A (Life 793 Technologies) supplemented with $1 \mu \mathrm{g} / \mathrm{ml}$ of Heparin (Sigma), 2mM L-Glutamine and 20ng/ml of EGF 794 and FGF-basic. Cells were cultured as a neurospheres in tissue culture flasks. When they were cultured 795 as an adherent culture, flask was additionally coated with Laminin (L2020, Sigma) for EPD210FH cells and with Geltrex (A1569601, Life Technologies) for EP1NS cells. Pediatric patient-derived SUpcGBM2 cells were cultivated as neurospheres as previously described. (Katrin Schramm paper, 2019). All cells were routinely tested free of mycoplasma contamination and authenticated by Single 
799 Nucleotide Polymorphism profiling (Multiplexion $\mathrm{GmbH}$ ). All cell models were grown at $37^{\circ} \mathrm{C}$ with

$800 \quad 5 \% \mathrm{CO} 2$.

801

\section{Lentiviral Transduction}

803 shRNA plasmids and sgRNA plasmids were constructed as previously described (STK3 paper). All 804 oligos are ordered from Sigma. Target sequences of all oligos are listed in Supplementary Table S8. 805 Transduction was performed in the presence of protamine sulfate (final concentration $5 \mathrm{ug} / \mathrm{mL}$, Sigma806 Aldrich) Transduced cells were further cultured and GFP signal was analyzed by BD FACS Canto for 807 GFP expression. Reduction of the percentage of GFP-positive cells indicates that the infected cells 808 expressing a particular shRNA/ sgRNA have a growth disadvantage in comparison to the non-infected 809 cells.

\section{Drug treatments}

812 All drugs were prepared according to protocols provided by company (Supplementary Table S9) Cells were seeded into 96-well cell culture treated plates at a density of 5000 cells in $100 \mu 1$ respected medium per well. On the next day morning, cells were treated with increasing concentrations ( $200 \mathrm{nM}$, $400 \mathrm{nM}, 800 \mathrm{nM}, 1.6 \mu \mathrm{M}, 3.2 \mu \mathrm{M}, 6.4 \mu \mathrm{M}$ and $12.8 \mu \mathrm{M}$ ) of each drug or equivalent dilutions of solvent. Cell viability was assessed after 72 hours using the CellTiter-Glo ${ }^{\circledR}$ luminescent cell viability assay (Promega) and an automated plate reader Mythras. All samples were assayed in triplicates and normalized to the average values of the corresponding mock control on the same plate and analyzed

\section{Western blot analysis}

822 For knockdown and/or knockout studies, cells were infected as described above and cultured for either

8234 days (knockdown) or 5 days (knockout). Before harvesting, cells were washed with phosphate 824 buffered saline (PBS) and collected as pellets. Then, pellets were lysed in RIPA buffer (Sigma825 Aldrich) supplemented with protease and phosphatase inhibitors for 30 minutes on ice. After centrifugation at $13,000 \mathrm{rpm}$ for 10 minutes at $4{ }^{\circ} \mathrm{C}$, the supernatants were collected and protein concentrations were determined using a Bicinchoninic acid (BCA) assay (Sigma-Aldrich) with 828 PierceTM bovine serum albumin standards (Thermo Fisher). Lysates were mixed with NuPAGE® 829 LSD Sample buffer (Life Technologies) supplemented with $10 \%$ 2-mercaptoethanol and denatured 830 for 5 minutes at $95^{\circ} \mathrm{C}$. Afterwards they were subjected to sodium dodecyl sulfate-polyacrylamide gel 831 electrophoresis according to standard procedures using 4-12\% Bis-Tris gels and afterwards transferred 832 to polyvinylidene difluoride membranes. Membranes were incubated with respective primary 
antibodies at $4{ }^{\circ} \mathrm{C}$ overnight (Supplementary Table S10). Horseradish peroxidase-conjugated anti-

834 rabbit (1:5,000, Santa Cruz, sc-2054) secondary antibody were applied for 1 hour at room temperature and chemiluminescent detection was carried out using AmershamTM ECLTM or ECLTM Prime

836 Western Blotting detection reagents (GE Healthcare). The same membranes were stripped with 837 stripping buffer (Sigma) according to protocol and incubated with conjugated beta-actin antibody as a 838 loading control.

840 Data availability

841 The novel sequencing data raw materials (HiC, CTCF, WGBS) will be included into the European 842 Genome-phenome archive (https://www.ebi.ac.uk/ega/home) under the accession number: 843 GAS00001002696; this source already contains other data types (RNA-seq, H3K27ac) for the 844 corresponding target tumor samples.

846 Code availability

847 Scripts for processing the raw data and generating figures can be obtained upon request.

\section{References}

8501 Pajtler, K. W. et al. Molecular Classification of Ependymal Tumors across All CNS 851 Compartments, Histopathological Grades, and Age Groups. Cancer Cell 27, 728-743, 852 doi:10.1016/j.ccell.2015.04.002 (2015).

8532 Pui, C. H., Gajjar, A. J., Kane, J. R., Qaddoumi, I. A. \& Pappo, A. S. Challenging issues in 854 pediatric oncology. Nat Rev Clin Oncol 8, 540-549, doi:10.1038/nrclinonc.2011.95 (2011). 8553 Siegel, R. L., Miller, K. D. \& Jemal, A. Cancer statistics, 2016. CA Cancer J Clin 66, 7-30, 856 doi:10.3322/caac.21332 (2016).

8574 Downing, J. R. et al. The Pediatric Cancer Genome Project. Nat Genet 44, 619-622, 858 doi:10.1038/ng.2287 (2012).

8595 Mack, S. C. et al. Epigenomic alterations define lethal CIMP-positive ependymomas of 860 infancy. Nature 506, 445-450, doi:10.1038/nature13108 (2014).

8616 Witt, H. et al. Delineation of two clinically and molecularly distinct subgroups of posterior 862 fossa ependymoma. Cancer Cell 20, 143-157, doi:10.1016/j.ccr.2011.07.007 (2011).

8637 Parker, M. et al. C11orf95-RELA fusions drive oncogenic NF-kappaB signalling in 864 ependymoma. Nature 506, 451-455, doi:10.1038/nature13109 (2014). 
8658 Pajtler, K. W. et al. YAP1 subgroup supratentorial ependymoma requires TEAD and nuclear factor I-mediated transcriptional programmes for tumorigenesis. Nature communications $\mathbf{1 0}$, $1-16(2019)$.

8689 Panwalkar, P. et al. Immunohistochemical analysis of H3K27me3 demonstrates global reduction in group-A childhood posterior fossa ependymoma and is a powerful predictor of outcome. Acta Neuropathol 134, 705-714, doi:10.1007/s00401-017-1752-4 (2017).

Pajtler, K. W. et al. Molecular heterogeneity and CXorf67 alterations in posterior fossa group A (PFA) ependymomas. Acta Neuropathol 136, 211-226, doi:10.1007/s00401-018-1877-0 (2018).

Hubner, J. M. et al. EZHIP / CXorf67 mimics K27M mutated oncohistones and functions as an intrinsic inhibitor of PRC2 function in aggressive posterior fossa ependymoma. Neuro Oncol, doi:10.1093/neuonc/noz058 (2019).

Araki, A. et al. Chromosome 1q gain and tenascin-C expression are candidate markers to define different risk groups in pediatric posterior fossa ependymoma. Acta neuropathologica communications 4, 88 (2016).

Grubert, F. et al. Landscape of cohesin-mediated chromatin loops in the human genome. Nature 583, 737-743 (2020).

Rhie, S. K. et al. A high-resolution 3D epigenomic map reveals insights into the creation of the prostate cancer transcriptome. Nature communications 10, 1-12 (2019). Kloetgen, A. et al. Three-dimensional chromatin landscapes in T cell acute lymphoblastic leukemia. Nature Genetics 52, 388-400 (2020).

17 Capper, D. et al. DNA methylation-based classification of central nervous system tumours. Nature 555, 469-474 (2018). profiling. Nature 553, 101-105, doi:10.1038/nature25169 (2018).

19 Dixon, J. R. et al. Integrative detection and analysis of structural variation in cancer genomes. Nat Genet 50, 1388-1398, doi:10.1038/s41588-018-0195-8 (2018).

Lupianez, D. G. et al. Disruptions of topological chromatin domains cause pathogenic rewiring of gene-enhancer interactions. Cell 161, 1012-1025, doi:10.1016/j.cell.2015.04.004 (2015).

21 Chakraborty, A. \& Ay, F. Identification of copy number variations and translocations in cancer cells from Hi-C data. Bioinformatics 34, 338-345 (2018). 
22 Wang, Y. et al. LSD1 co-repressor Rcor2 orchestrates neurogenesis in the developing mouse brain. Nature communications 7, 10481, doi:10.1038/ncomms10481 (2016). Maes, T. et al. ORY-1001, a potent and selective covalent KDM1A inhibitor, for the treatment of acute leukemia. Cancer Cell 33, 495-511. e412 (2018). Rajarajan, P. et al. Neuron-specific signatures in the chromosomal connectome associated with schizophrenia risk. Science (New York, N.Y.) 362, doi:10.1126/science.aat4311 (2018). Davis, C. A. et al. The Encyclopedia of DNA elements (ENCODE): data portal update. Nucleic acids research 46, D794-D801, doi:10.1093/nar/gkx1081 (2018). Michealraj, K. A. et al. Metabolic Regulation of the Epigenome Drives Lethal Infantile Ependymoma. Cell, doi:10.1016/j.cell.2020.04.047 (2020). Mechanisms of Temozolomide Sensitivity in Glioblastoma Stem Cells. Cell Rep 27, 971-986 e979, doi:10.1016/j.celrep.2019.03.047 (2019).

Flavahan, W. A. et al. Insulator dysfunction and oncogene activation in IDH mutant gliomas. Nature 529, 110-114, doi:10.1038/nature16490 (2016).

Flavahan, W. A. et al. Altered chromosomal topology drives oncogenic programs in SDHdeficient GISTs. Nature 575, 229-233, doi:10.1038/s41586-019-1668-3 (2019).

Fujii, S., Matsumoto, S., Nojima, S., Morii, E. \& Kikuchi, A. Arl4c expression in colorectal and lung cancers promotes tumorigenesis and may represent a novel therapeutic target. Oncogene 34, 4834-4844, doi:10.1038/onc.2014.402 (2015).

Takagi, S. et al. LSD1 inhibitor T-3775440 inhibits SCLC cell proliferation by disrupting LSD1 interactions with SNAG domain proteins INSM1 and GFI1B. Cancer Research 77, 4652-4662 (2017).

32 Milde, T. et al. A novel human high-risk ependymoma stem cell model reveals the differentiation-inducing potential of the histone deacetylase inhibitor Vorinostat. Acta neuropathologica 122, 637 (2011).

33 Park, D. E. et al. Merkel cell polyomavirus activates LSD1-mediated blockade of noncanonical BAF to regulate transformation and tumorigenesis. Nature Cell Biology 22, 603615 (2020).

4 Lathia, J. D. et al. Integrin alpha 6 regulates glioblastoma stem cells. Cell Stem Cell 6, 421 432, doi:10.1016/j.stem.2010.02.018 (2010).

Martin, T. A. \& Jiang, W. G. Evaluation of the expression of stem cell markers in human breast cancer reveals a correlation with clinical progression and metastatic disease in ductal carcinoma. Oncol Rep 31, 262-272, doi:10.3892/or.2013.2813 (2014). 
Hoogland, A. M. et al. Validation of stem cell markers in clinical prostate cancer: alpha6integrin is predictive for non-aggressive disease. Prostate 74, 488-496, doi:10.1002/pros.22768 (2014). Schober, M. \& Fuchs, E. Tumor-initiating stem cells of squamous cell carcinomas and their control by TGF-beta and integrin/focal adhesion kinase (FAK) signaling. Proceedings of the National Academy of Sciences of the United States of America 108, 10544-10549, doi:10.1073/pnas.1107807108 (2011).

Haraguchi, N. et al. CD49f-positive cell population efficiently enriches colon cancerinitiating cells. Int J Oncol 43, 425-430, doi:10.3892/ijo.2013.1955 (2013).

Zhang, Y. et al. Overexpression of LAMC1 predicts poor prognosis and enhances tumor cell invasion and migration in hepatocellular carcinoma. J Cancer 8, 2992-3000, doi:10.7150/jca.21038 (2017).

Consortium, E. P. An integrated encyclopedia of DNA elements in the human genome. Nature 489, 57-74 (2012).

41 Schmitt, A. D. et al. A compendium of chromatin contact maps reveals spatially active regions in the human genome. Cell reports 17, 2042-2059 (2016).

Rao, S. S. et al. A 3D map of the human genome at kilobase resolution reveals principles of chromatin looping. Cell 159, 1665-1680 (2014).

43 Servant, N. et al. HiC-Pro: an optimized and flexible pipeline for Hi-C data processing. Genome biology 16, 259 (2015).

Durand, N. C. et al. Juicebox provides a visualization system for Hi-C contact maps with unlimited zoom. Cell systems 3, 99-101 (2016).

Kaul, A., Bhattacharyya, S. \& Ay, F. Identifying statistically significant chromatin contacts from Hi-C data with FitHiC2. Nature Protocols 15, 991-1012 (2020).

Shin, H. et al. TopDom: an efficient and deterministic method for identifying topological domains in genomes. Nucleic acids research 44, e70-e70 (2016).

Durand, N. C. et al. Juicer Provides a One-Click System for Analyzing Loop-Resolution HiC Experiments. Cell systems 3, 95-98, doi:10.1016/j.cels.2016.07.002 (2016).

4 Lieberman-Aiden, E. et al. Comprehensive mapping of long-range interactions reveals folding principles of the human genome. Science (New York, N.Y.) 326, 289-293, doi:10.1126/science.1181369 (2009).

Okonechnikov, K., Erkek, S., Korbel, J. O., Pfister, S. M. \& Chavez, L. InTAD: chromosome conformation guided analysis of enhancer target genes. BMC bioinformatics 20, 60 (2019). 
$96650 \quad$ Sherman, B. T. \& Lempicki, R. A. Systematic and integrative analysis of large gene lists 967 using DAVID bioinformatics resources. Nature protocols 4, 44 (2009).

96851 Dixon, J. R. et al. Topological domains in mammalian genomes identified by analysis of 969 chromatin interactions. Nature 485, 376-380 (2012).

97052 Li, H. \& Durbin, R. Fast and accurate short read alignment with Burrows-Wheeler transform. 971 bioinformatics 25, 1754-1760 (2009).

97253 Zhang, Y. et al. Model-based analysis of ChIP-Seq (MACS). Genome biology 9, 1-9 (2008).

97354 Ross-Innes, C. S. et al. Differential oestrogen receptor binding is associated with clinical 974 outcome in breast cancer. Nature 481, 389-393 (2012).

97555 Hovestadt, V. et al. Decoding the regulatory landscape of medulloblastoma using DNA 976 methylation sequencing. Nature 510, 537-541 (2014).

$97756 \quad J u ̈ h l i n g$, F. et al. metilene: Fast and sensitive calling of differentially methylated regions from 978 bisulfite sequencing data. Genome research 26, 256-262 (2016). 
Figures

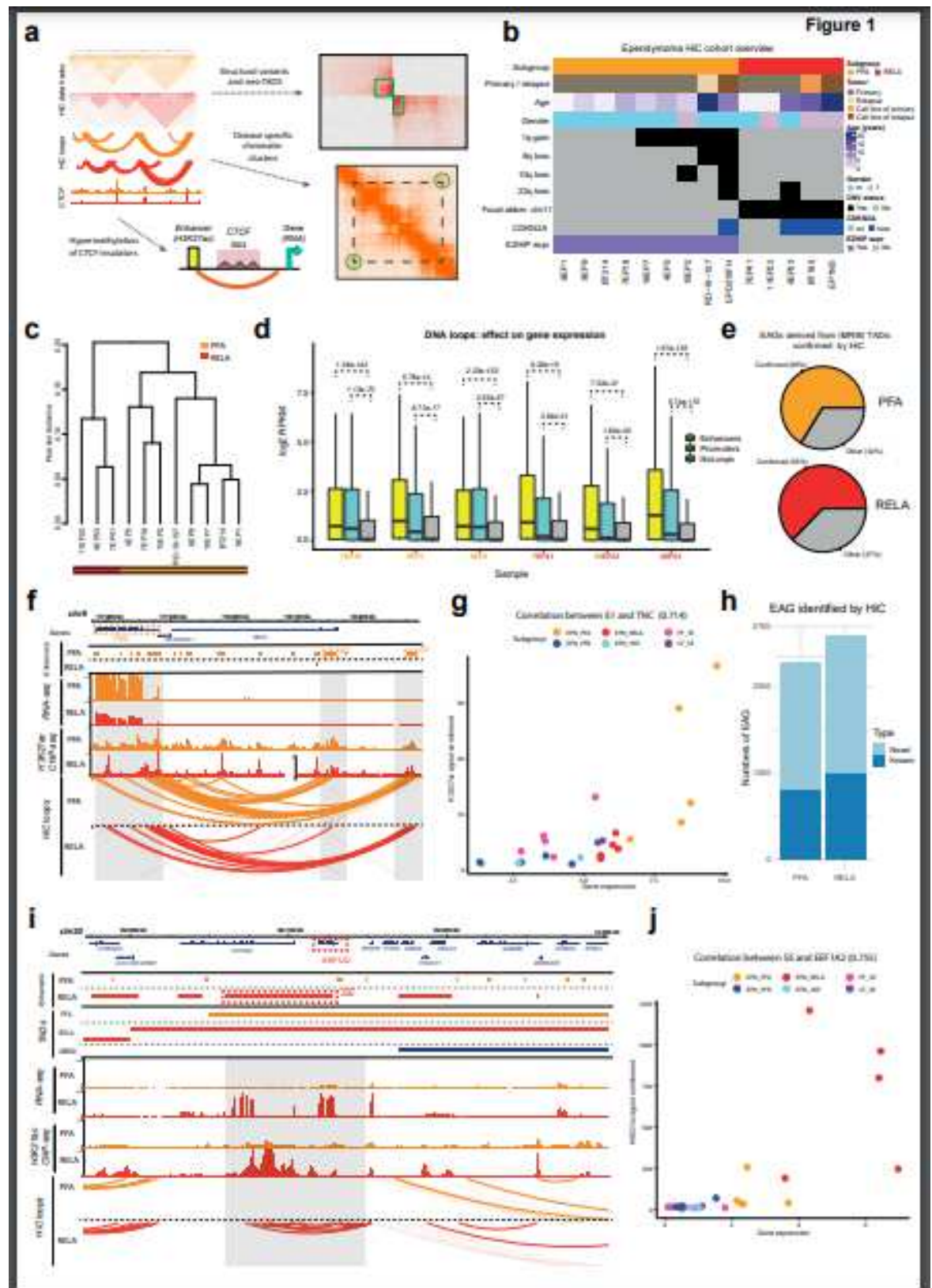

Figure 1

[Please see the manuscript file to view the figure caption.] 


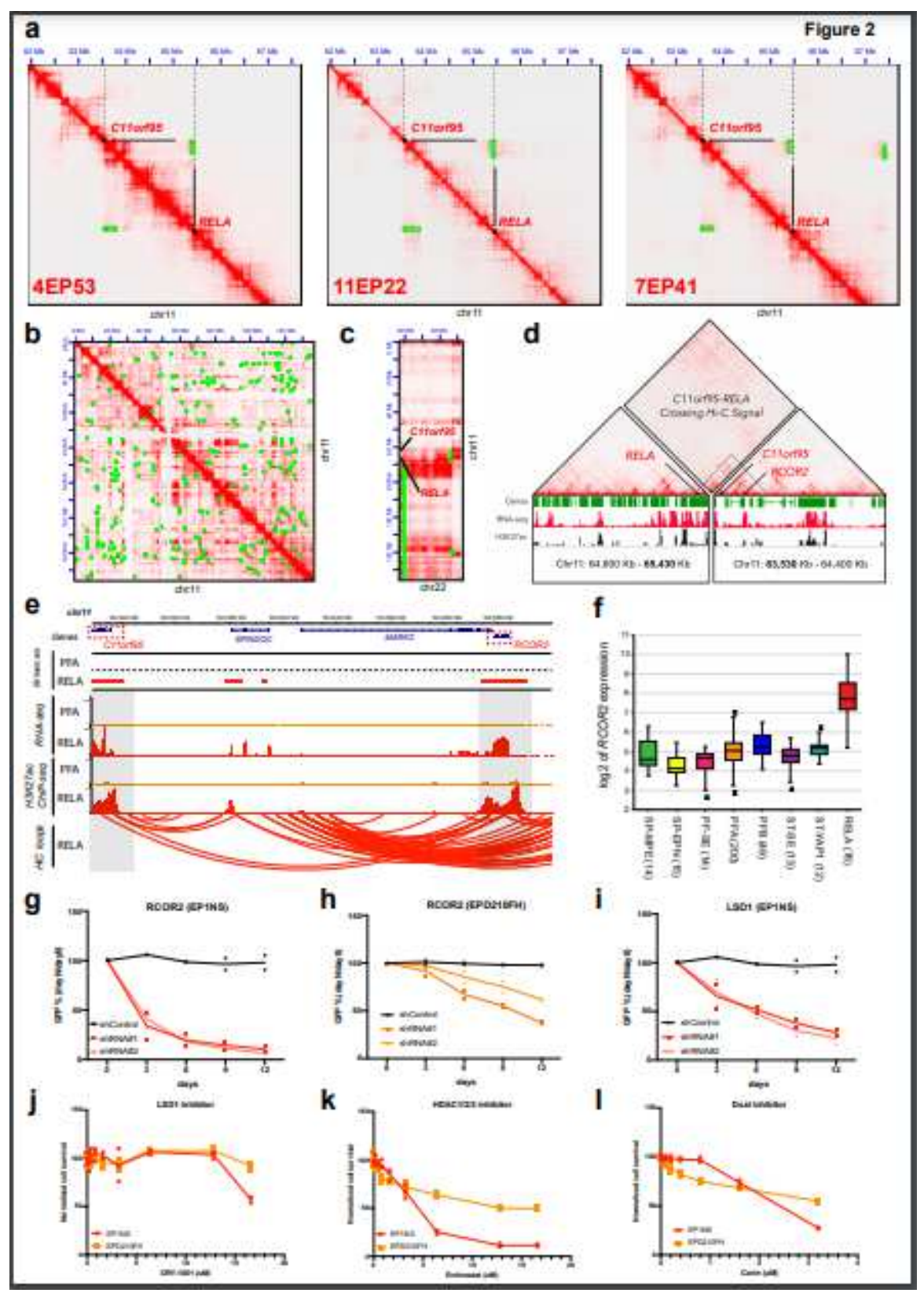

Figure 2

[Please see the manuscript file to view the figure caption.] 


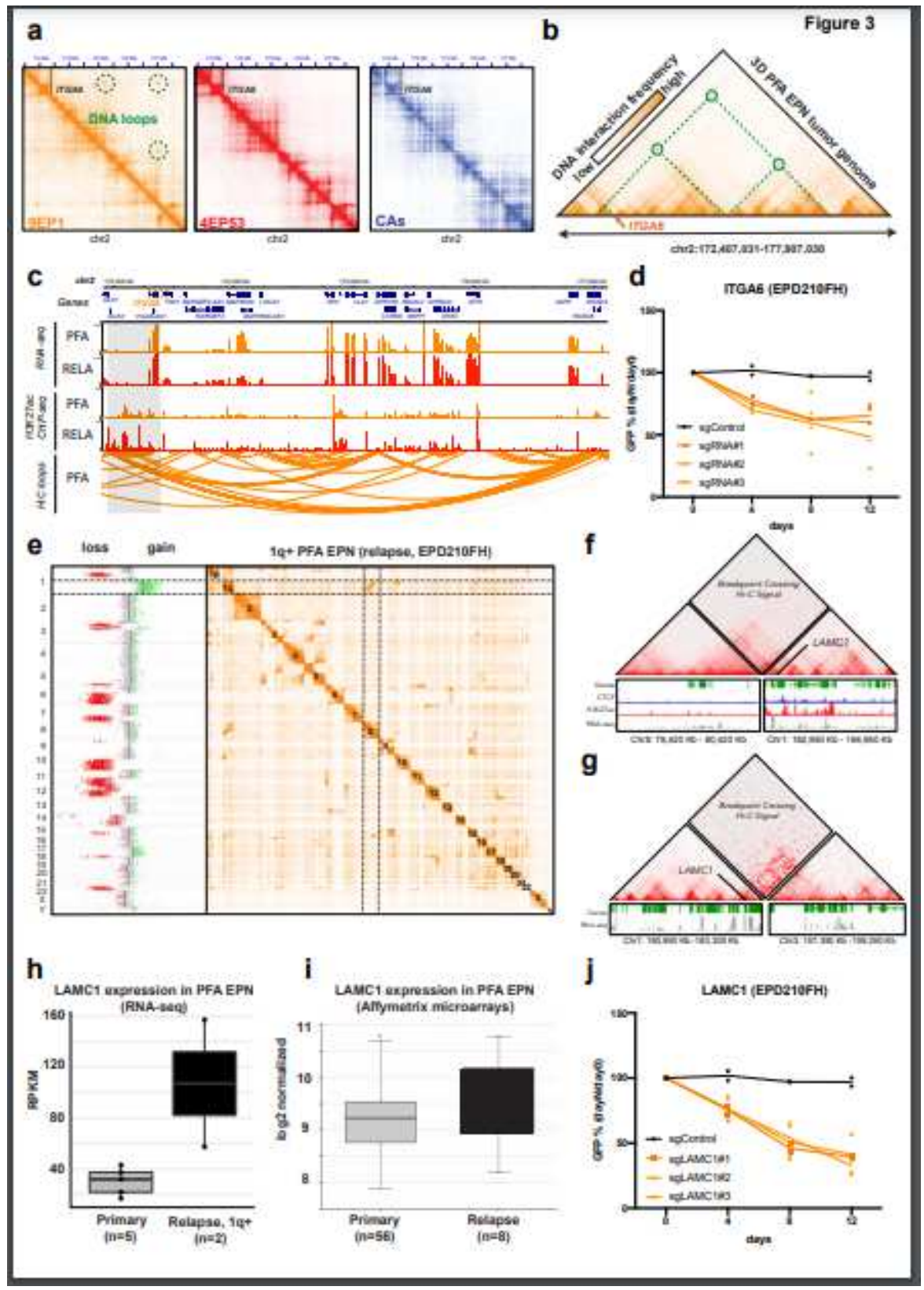

Figure 3

[Please see the manuscript file to view the figure caption.] 


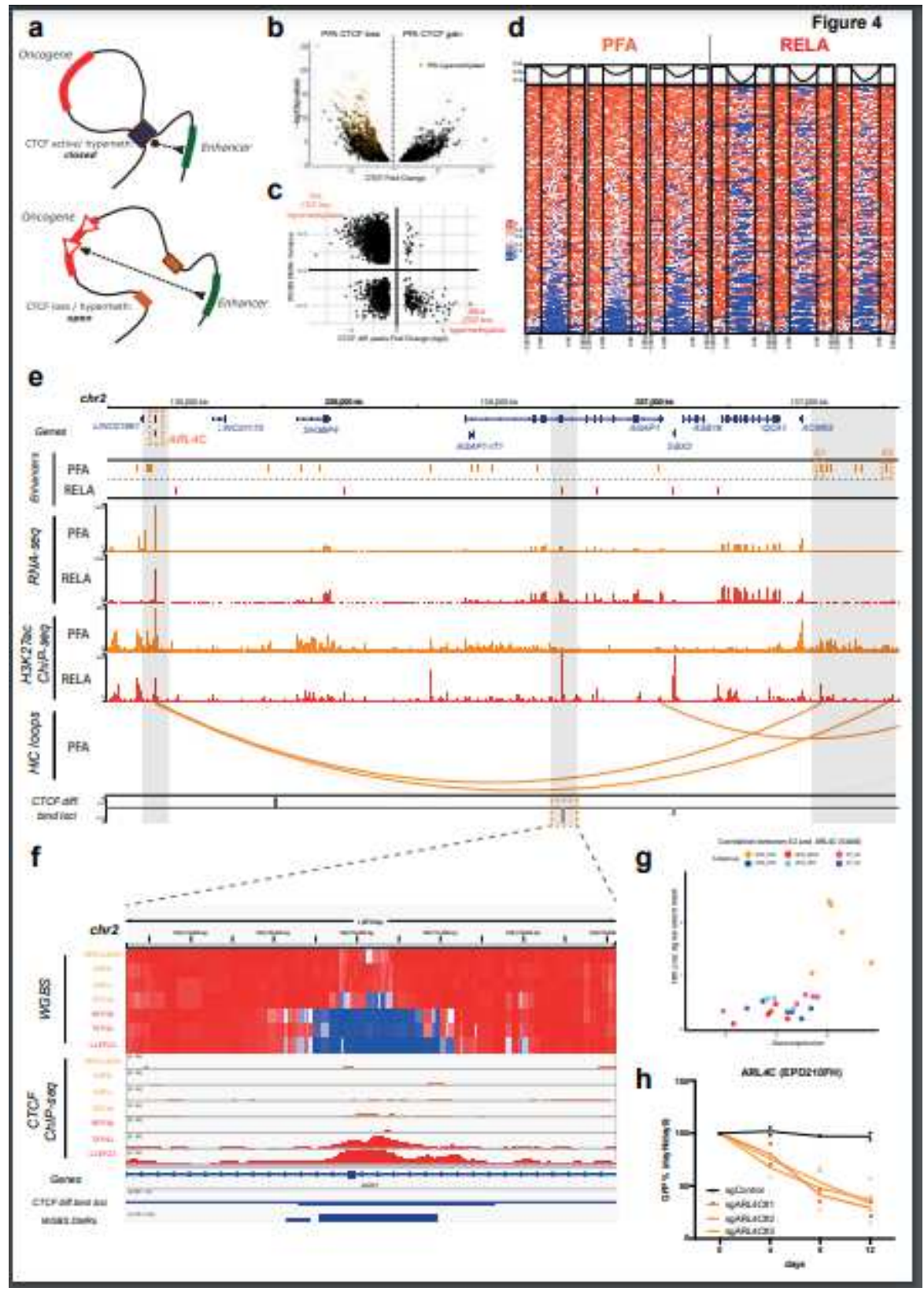

Figure 4

[Please see the manuscript file to view the figure caption.]

\section{Supplementary Files}

This is a list of supplementary files associated with this preprint. Click to download.

- EPNHiCtables.011020.xlsx

- SuppFigure1041020.pdf

- SuppFigure2041020.pdf

- SuppFigure3041020.pdf

- SuppFigure4041020.pdf 